TAIWANESE JOURNAL OF MATHEMATICS

Vol. 15, No. 5, pp. 2059-2080, October 2011

This paper is available online at http://tjm.math.ntu.edu.tw/index.php/TJM

\title{
DISCONTINUOUS GENERALIZED QUASI-VARIATIONAL INEQUALITIES WITH APPLICATION TO FIXED POINTS
}

\author{
Paolo Cubiotti and Jen-Chih Yao*
}

\begin{abstract}
We consider the following generalized quasi-variational inequality problem introduced in [7]: given a real normed space $X$ with topological dual $X^{*}$, two sets $C, D \subseteq X$ and two multifunctions $S: C \rightarrow 2^{D}$ and $T: C \rightarrow 2^{X^{*}}$, find $(\hat{x}, \hat{\varphi}) \in C \times X^{*}$ such that

$$
\hat{x} \in S(\hat{x}), \quad \hat{\varphi} \in T(\hat{x}) \quad \text { and } \quad\langle\hat{\varphi}, \hat{x}-y\rangle \leq 0 \quad \text { for all } \quad y \in S(\hat{x}) .
$$
\end{abstract}

We prove an existence theorem where $T$ is not assumed to have any continuity or monotonicity property, improving some aspects of the main result of [7]. In particular, the coercivity assumption is meaningfully weakened. As an application, we prove a theorem of the alternative for the fixed points of a Hausdorff lower semicontinuous multifunction. In particular, we obtain sufficient conditions for the existence of a fixed point which belongs to the relative boundary of the corresponding value.

\section{INTRODUCTION}

Given a real topological vector space $X$ with topological dual $X^{*}$, a closed convex set $C \subseteq X$, and two multifunctions $S: C \rightarrow 2^{C}$ and $T: C \rightarrow 2^{X^{*}}$, the classical generalized quasi-variational inequality problem associated with $C$, $T$ and $S$ (which will be shortly denoted by $\operatorname{GQVI}(C, T, S)$ ) is to find a pair $(\hat{x}, \hat{\varphi}) \in C \times X^{*}$ such that

$$
\hat{x} \in S(\hat{x}), \quad \hat{\varphi} \in T(\hat{x}) \quad \text { and } \quad\langle\hat{\varphi}, \hat{x}-y\rangle \leq 0 \quad \text { for all } y \in S(\hat{x}) .
$$

The problem $\operatorname{GQVI}(C, T, S)$ was firstly introduced in finite-dimensional setting by Chan and Pang [6], while, in the above infinite-dimensional form, it was firstly considered by Shih and Tan [31].

Received May 11, 2010, accepted August 20, 2010.

Communicated by Mau-Hsiang Shih.

2010 Mathematics Subject Classification: 90C29, 49J40.

Key words and phrases: Generalized quasi-variational inequalities, Affine hull, Lower semicontinuity, Hausdorff lower semicontinuity, Fixed points, Relative interior, Relative boundary.

*Corresponding author. 
When the multifunction $T$ is single-valued, the prefix "generalized" is usually omitted. In this latter form, it was firstly studied by Bensoussans and Lions in connection with impulse control theory [3-5].

When $T$ is single-valued and the multifunction $S$ is identically equal to the set $C$, the problem $\operatorname{GQVI}(C, T, S)$ reduces to the classical variational inequality problem [23].

During the last decades, the existence of solutions for the problem $\operatorname{GQVI}(C$, $T, S$ ) has been widely investigated, due to the wide range of applications of the variational inequality theory (including mechanics, game theory, complementarity problems, control theory, network equilibrium and so on; see, for instance [2, 16$18,20-22,27,33]$ and the references therein).

In the last years, moreover, a great effort has been made to investigate the case where the multifunction $T$ is not assumed to have any continuity or monotonicity property, going out from these two typical research lines. In this direction, we refer to the papers $[8,9,11-15,26,29,32,34-37]$ and to references therein. In particular, we refer to the paper $[26,35]$ for discussions and comparisons about the basic regularity assumptions imposed on $T$.

Very recently, in the paper [7], Chu and Lin have considered the case where the multifunction $S$ can take its values outside the set $C$. More precisely, given the sets $X$ and $C$ and the multifunction $T$ as above, and given a closed convex set $D \subseteq X$ and a multifunction $S: C \rightarrow 2^{D}$, they considered the following extended generalized quasi-variational inequality problem associated with $C, D, T$ and $S$ :

$\operatorname{EGQVI}(C, D, T, S)$ : find $(\hat{x}, \hat{\varphi}) \in C \times X^{*}$ such that condition (1) holds.

It is worth noticing that condition (1) implies in particular that $C \cap D \neq \emptyset$, since $\hat{x} \in C$ and $S(\hat{x}) \subseteq D$ (as it is correctly remarked in [7], it is not restrictive to assume that $C \subseteq D$ ). It should be observed that the above generalization brings further technical problems while trying to investigate existence results. Indeed, the fact that $S$ takes its values in the whole set $D$ makes some of the known techniques not applicable.

In the paper [7], the authors prove an existence result for the problem $\operatorname{EGQVI}(C$, $D, T, S$ ) associated with a non-monotone and discontinuous multifunction $T$, in the spirit of the papers $[11,12,13]$. The following is their result (where "aff(D)" denotes the affine hull of the set $D$ ).

Theorem 1.1. (Theorem 2.7 of [7]). Let $\left(X,\|\cdot\|_{X}\right)$ be a real normed space, $C$ and $D$ two closed convex subsets of $X$, and let $T: X \rightarrow 2^{X^{*}}$ and $S: C \rightarrow 2^{D}$ two multifunctions. Let $H$ and $K$ be two nonempty compact subsets of $C$, such that $H \subseteq K$ and $H$ is finite-dimensional. Assume that:

(i) $S(x) \cap H \neq \emptyset$ for all $x \in C$;

(ii) the multifunction $S$ is Hausdorff continuous with closed convex values; 
(iii) $\operatorname{int}_{\mathrm{aff}(\mathrm{D})}(S(x)) \neq \emptyset$ for all $x \in C$;

(iv) $T(x)$ is nonempty, convex and weakly-star compact for each $x \in D$;

(v) for each $y \in D$, the set $\left\{x \in D: \inf _{\varphi \in T(x)}\langle\varphi, x-y\rangle \leq 0\right\}$ is closed;

(vi) $T$ and $S$ satisfy the generalized $V_{0}$-Karamardian condition on $(D, H, K)$ for some neighborhood $V_{0}$ of the origin in $X$ : for each $x \in\left(D+V_{0}\right) \backslash K$ and each $\varphi \in T(x)$, one has

$$
\sup _{y \in S(x) \cap H}\langle\varphi, x-y\rangle>0 .
$$

Then there exists $(\hat{x}, \hat{\varphi}) \in K \times X^{*}$ which solves $\operatorname{EGQVI}(C, D, T, S)$.

Before going on, we remark that the regularity condition (v) on the multifunction $T$ does not imply any kind of continuity for $T$ over the set $D$ (see $[12,13$, 35]). Moreover (see [35]), it is independent (although similar) from the analogous assumption on $T$ made in $[8,9,11,29,30]$.

If we compare Theorem 1.1 with similar results (see $[8,9,11-13,15,26,30$, $36,37]$ and references therein), it appears clearly that the possibility for $S$ to take its values in the whole set $D$ is obtained via the heavy coercivity condition (vi). Indeed, the spirit of coercivity conditions of Karamardian's type is to force possible solutions of the GQVI problem to stay in the compact set $K$. Consequently, it seems reasonable that it should be required only for the fixed points of $S$ (again, see [9, $11-13,15,26,30,36,37])$. Moreover, such a requirement is very important in view of possible applications, as showed in [10].

In Theorem 1.1, conversely, the coercivity condition (vi) applies not only to all points of $D$ (without considering if they are fixed points of $S$ or not), but also to all points of $D+V_{0}$ ( $V_{0}$ being a suitable neighborhood of the origin). This forces, in particular, the multifunction $T$ to be defined over the whole space $X$ (and not only over $C$ ).

The aim of this paper is to prove an existence result for the problem $\operatorname{EGQVI}(C$, $D, T, S$ ) where, while retaining the basic regularity assumptions (i)-(v) on the multifunctions $T$ and $S$ as in Theorem 1.1, we replace the generalized $V_{0}$-Karamardian condition (vi) by the following weaker coercivity condition:

(A) for each $x \in C \backslash K$, with $x \in S(x)$, and each $\varphi \in T(x)$, one has

$$
\sup _{y \in S(x) \cap H}\langle\varphi, x-y\rangle>0 .
$$

Beyond the pure theoretic generality, it is quite easy to check how condition (A) is really more manageable than assumption (vi) of Theorem 1.1, since it can be satisfied by assuming "natural" conditions over the data. Indeed, assumption (A) is satified, for instance, if the multifunction $S$ satisfies assumption (i) of Theorem 1.1 
and its fixed-point set $\operatorname{Fix}(S):=\{x \in C: x \in S(x)\}$ (which is closed by (ii)) is compact. In this occurrence, one can choose $K:=H \cup \operatorname{Fix}(S)$, and condition (A) is automatically satisfied since there is no fixed point of $S$ outside the set $K$. We shall return on this fact later.

The result we want to prove, in its full generality, is the following (in what follows, we shall put $\left.S_{C}(x):=S(x) \cap C\right)$.

Theorem 1.2. Let $\left(X,\|\cdot\|_{X}\right)$ be a real Banach space with topological dual $X^{*}, C \subseteq D \subseteq X$ closed convex sets, and let $H \subseteq K$ be two compact subsets of $C$, where $H$ is finite-dimensional. Let $S: C \rightarrow 2^{D}$ and $T: C \rightarrow 2^{X^{*}}$ be two multifunctions. Assume that:

(i) $S(x) \cap H \neq \emptyset$ for all $x \in C$;

(ii) the multifunction $S$ is Hausdorfflower semicontinuous with closed graph and convex values;

(iii) $\operatorname{int}_{\mathrm{aff}(\mathrm{D})}(S(x) \cap C) \neq \emptyset$ for all $x \in C$;

(iv) $T(x)$ is nonempty and weakly-star compact for each $x \in C$, and convex for each $x \in C$, with $x \in S(x)$;

(v) for each $y \in C$, the set $\left\{x \in C: \inf _{\varphi \in T(x)}\langle\varphi, x-y\rangle \leq 0\right\}$ is compactly closed;

(vi) for each $x \in C \backslash K$, with $x \in S(x)$, and each $\varphi \in T(x)$, one has

$$
\sup _{y \in S(x) \cap H}\langle\varphi, x-y\rangle>0 .
$$

Then there exists $(\hat{x}, \hat{\varphi}) \in K \times X^{*}$ which solves $\operatorname{GQVI}\left(C, T, S_{C}\right)$. Moreover, if $\hat{x} \in \operatorname{int}_{\mathrm{aff}(\mathrm{D})}(C)$, then the pair $(\hat{x}, \hat{\varphi})$ solves $\operatorname{EGQVI}(C, D, T, S)$.

Theorem 1.2 will be proved in Section 3, while in Section 2 we will fix some notations and preliminaries. In Section 4, starting from the above remarks, we shall derive some corollaries and consequences of Theorem 1.2. As an application, we shall prove a theorem of the alternative for the fixed points of a Hausdorff lower semicontinuos multifunction $S$ (Theorem 4.3), which extends to infinite-dimensional setting a recent result of the author (Theorem 3.1 of [10]). In particular, our result admits the following corollary, which gives sufficient conditions for the existence of a fixed point $\hat{x}$ which lies on the relative boundary of the corresponding value $S(\hat{x})$ (that is, the boundary of $S(\hat{x})$ in its affine hull).

Theorem 1.3. Let $X$ be a real separable Hilbert space with scalar product $\langle\cdot, \cdot\rangle$, let $C \subseteq X$ be a closed convex set which contains more than one point, and whose affine hull aff(C) is closed in $X$, and let $S: C \rightarrow 2^{C}$ be a multifunction. Assume that: 
(i) the multifunction $S$ is Hausdorff lower semicontinuous with closed graph and convex values;

(ii) $\operatorname{int}_{\mathrm{aff}(\mathrm{C})}(S(x)) \neq \emptyset$ for all $x \in C$;

(iii) there exists a finite-dimensional compact set $H \subseteq C$ such that $S(x) \cap H \neq \emptyset$ for all $x \in C$.

Then, at least one of the following assertions holds:

(A) The set $\operatorname{Fix}(S):=\{x \in C: x \in S(x)\}$ is not totally bounded.

(B) There exists $\hat{x} \in C$ such that $\hat{x} \in \partial_{r} S(\hat{x})$.

Before ending this section, we remark that in the proof of Theorem 1.2 we employ a technical construction which is deeply different from the one used in [7].

\section{Preliminaries}

Let $U$ and $V$ be topological spaces. A multifunction $F: U \rightarrow 2^{V}$ is said to be lower semicontinuos [resp., upper semicontinuous] at $x_{0} \in U$ if for each open set $A \subseteq V$, with $F\left(x_{0}\right) \cap A \neq \emptyset$ [resp., with $F\left(x_{0}\right) \subseteq A$ ], there exists a neighborhood $W$ of $x_{0}$ in $U$ such that

$$
\begin{array}{llll} 
& F(x) \cap A \neq \emptyset & \text { for all } & x \in W \\
\text { [resp., } & F(x) \subseteq A & \text { for all } & x \in W] .
\end{array}
$$

We say that $F$ is lower [resp., upper] semicontinuous in $U$ if it is lower [resp., upper] semicontinuous at each point $x \in U$. The graph of $F$ is the set

$$
\operatorname{Gr}(F):=\{(x, y) \in U \times V: y \in F(x)\} .
$$

We recall (see [24]) that if $G r(F)$ is closed, then each set $F(x)$ is closed; if $F$ is upper semicontinuos in $U$ with closed values, then $\operatorname{Gr}(F)$ is closed; if $\operatorname{Gr}(F)$ is closed and $V$ is compact, then $F$ is upper semicontinuous. A selection of the multifunction $F$ is a (single-valued) function $f: U \rightarrow V$ such that $f(x) \in F(x)$ for all $x \in U$.

Let $\left(X,\|\cdot\|_{X}\right)$ be a normed space (whose origin will be denoted by $0_{X}$ ). If $x_{0} \in X$ and $r>0$, we denote by $B\left(x_{0}, r\right)$ [resp., $\left.\bar{B}\left(x_{0}, r\right)\right]$ the open [resp., closed] ball in $X$ centered at $x_{0}$ with radius $r$.

If $A \subseteq X$, we denote by aff(A) and $\operatorname{span}(A)$, respectively, the affine hull and the linear hull of the set $A$. If $A \subseteq B \subseteq X$, we denote by $\operatorname{int}_{B}(A)$ and $\partial_{B} A$, respectively, the interior and the boundary of $A$ in $B$. Finally, we denote by $\operatorname{ri}(A)$ and $\partial_{r} A$, respectively, the relative interior and the relative boundary of the set $A$. That is, we put

$$
\operatorname{ri}(A):=\operatorname{int}_{\mathrm{aff}(\mathrm{A})}(A), \quad \partial_{r} A:=\partial_{\mathrm{aff}(\mathrm{A})} A .
$$


We say that the set $A \subseteq X$ is compactly closed if its intersection with each compact subset of $X$ is closed. Of course, each closed subset of $X$ is compactly closed. Finally, we denote by $\overline{\mathrm{co}}(A)$ the closed convex hull of the set $A$.

If $U$ is a topological space, a multifunction $F: U \rightarrow 2^{X}$ is said to be Hausdorfflower semicontinuos [resp., Hausdorff upper semicontinuous] at $x_{0} \in U$ if for each $\varepsilon>0$ there exists a neighborhood $W$ of $x_{0}$ in $U$ such that

$$
\begin{array}{llll} 
& F\left(x_{0}\right) \subseteq F(x)+B\left(0_{X}, \varepsilon\right) & \text { for all } & x \in W \\
\text { [resp., } & F(x) \subseteq F\left(x_{0}\right)+B\left(0_{X}, \varepsilon\right) & \text { for all } & x \in W] .
\end{array}
$$

As before, we say that $F$ is Hausdorff lower [resp., upper] semicontinuous in $U$ if it is Hausdorff lower [resp., upper] semicontinuous at each point $x \in U$. It is known that Hausdorff lower semicontinuity implies lower semicontinuity, while upper semicontinuity implies Hausdorff upper semicontinuity. The converse implications are true if each set $F(x)$ is nonempty and compact (see Theorem 7.1.14 of [24])

The following result, which is a particular case of Theorem 3.1"' of [28], will be a fundamental tool in the sequel.

Theorem 2.1. (Michael). Let $U$ be a metric space, $\left(X,\|\cdot\|_{X}\right)$ a separable Banach space, and $\Phi: U \rightarrow 2^{X}$ a lower semicontinuous multifunction whose values are convex and have nonempty interior. Then $\Phi$ admits a continuous selection.

If $(X,\langle\cdot, \cdot\rangle)$ a Hilbert space and $A \subseteq X$, we put

$$
A^{\perp}:=\{x \in X:\langle x, v\rangle=0 \quad \forall v \in A\} .
$$

We observe the following fact.

Proposition 2.2. Let $(X,\langle\cdot, \cdot\rangle)$ be a Hilbert space, $A \subseteq X$ a convex set with nonempty relative interior, and let $z \in X, x \in \operatorname{ri}(A)$ be such that

$$
\langle z, x-y\rangle \leq 0 \quad \text { for all } y \in A .
$$

Then $z \in(A-A)^{\perp}$.

Proof. Let $v, w \in A$. Then we have

$$
\operatorname{aff}(\mathrm{A})=\mathrm{v}+\operatorname{span}(\mathrm{A}-\mathrm{v})=\mathrm{v}+\mathrm{F},
$$

where we put $F:=\operatorname{span}(A-v)$. Since $x \in \operatorname{int}_{v+F}(A)$, we get

$$
x-v \in \operatorname{int}_{F}(A-v) .
$$

Consequently, there exists $\sigma>0$ such that

$$
x-v+\left[B\left(0_{X}, \sigma\right) \cap F\right] \subseteq A-v .
$$


Since $w-v \in F$, there exists $\mu>0$ such that

$$
x-v+\mu(w-v) \in A-v,
$$

hence $x+\mu(w-v) \in A$. By assumption, we get

$$
\langle z, x-(x+\mu(w-v))\rangle \leq 0,
$$

hence $\langle z, w-v\rangle \geq 0$. Since $v$ and $w$ were any points in $A$, the conclusion follows.

Finally, we recall the following result.

Proposition 2.3. [11]. Let $U$ be a topological space, $\left(X,\|\cdot\|_{X}\right)$ a real normed space, $M$ an affine manifold of $X$, and $F: U \rightarrow 2^{M}$ a Hausdorff lower semicontinuous multifunction with nonempty closed convex values. Let $x_{0} \in U$, $y_{0} \in \operatorname{int}_{M}\left(F\left(x_{0}\right)\right)$.

Then, there exists a neighborhood $Z$ of $x_{0}$ in $U$ such that

$$
y_{0} \in \operatorname{int}_{M}\left(\bigcap_{x \in Z} F(x)\right) .
$$

\section{Proof of Theorem 1.2}

For the reader's convenience, we shall divide the proof into steps.

Step 1. First of all, we observe that by Theorem 6 at p.416 of [19], the set $\overline{\mathrm{CO}} K$ is compact. Moreover, in what follows we shall put $M:=\operatorname{aff}(\mathrm{D})$.

Let $B_{0}$ be an open ball in $X$ such that $\overline{\mathrm{co}}(K) \subseteq B_{0}$, and let

$$
C_{0}:=C \cap \bar{B}_{0}
$$

(of course, $\bar{B}_{0}$ denotes the closure of $B_{0}$ in $X$ ). By assumptions, we have that

$$
H \subseteq K \subseteq \overline{\mathrm{co}}(K) \subseteq C_{0} .
$$

Let $S_{0}: C_{0} \rightarrow 2^{C_{0}}$ be the multifunction defined by putting, for each $x \in C_{0}$,

$$
S_{0}(x):=S(x) \cap C_{0} .
$$

We observe the following facts.

(i) $\prime S_{0}(x) \cap H \neq \emptyset$ for all $x \in C_{0}$ (this follows at once by(i), since $H \subseteq C_{0}$ ).

(ii)/ The multifunction $S_{0}$ is Hausdorff lower semicontinuous with convex values and closed graph. 
The fact that $S_{0}$ has convex values follows from the convexity of $C_{0}$ and of each set $S(x)$. Moreover, $S_{0}$ has closed graph by (ii). In order to prove that $S_{0}$ is Hausdorff lower semicontinuous, we first prove that

$$
\operatorname{int}_{M}\left[S(x) \cap C_{0}\right] \neq \emptyset, \quad \text { for all } \quad x \in C .
$$

To this aim, let $x \in C$ be fixed, and let $v$ be any point in $S(x) \cap H$ (which is nonempty by assumption (i)). By (iii), we have that

$$
\operatorname{int}_{M}[S(x) \cap C] \neq \emptyset \text {. }
$$

Choose any point $u \in \operatorname{int}_{M}[S(x) \cap C]$. Since $M \backslash B_{0} \neq \emptyset$ (recall that $B_{0}$ is bounded), the set $M \backslash B_{0}$ is closed in $M, H \subseteq B_{0}$ and $H$ is compact, we have that

$$
\rho:=\inf \left\{d\left(a, M \backslash B_{0}\right): a \in H\right\}>0
$$

By convexity, we have that

$$
\left.\left.u_{t}:=v+t(u-v) \in \operatorname{int}_{M}[S(x) \cap C], \quad \text { for all } t \in\right] 0,1\right] .
$$

Choose $\bar{t} \in] 0,1]$ in such a way that

$$
\left\|u_{\bar{t}}-v\right\|_{X}<\frac{\rho}{4}
$$

Since $u_{\bar{t}} \in \operatorname{int}_{M}[S(x) \cap C]$, there exists $\sigma>0$ such that

$$
B\left(u_{\bar{t}}, \sigma\right) \cap M \subseteq S(x) \cap C .
$$

Let

$$
\alpha:=\min \left\{\sigma, \frac{\rho}{4}\right\}
$$

Now, observe that

$$
B\left(u_{\bar{t}}, \alpha\right) \cap M \subseteq B_{0} \cap M .
$$

To prove (5), observe that if we choose any point $x \in B\left(u_{\bar{t}}, \alpha\right) \cap M$, we have that

$$
\|x-v\|_{X} \leq\left\|x-u_{\bar{t}}\right\|_{X}+\left\|u_{\bar{t}}-v\right\|_{X} \leq \frac{\rho}{4}+\frac{\rho}{4}=\frac{\rho}{2} .
$$

Since $v \in H$, (3) implies that $d\left(v, M \backslash B_{0}\right) \geq \rho$, hence $x \in M \cap B_{0}$, as desired.

By (4) and (5) it follows that

$$
B\left(u_{\bar{t}}, \alpha\right) \cap M \subseteq B_{0} \cap M \cap S(x) \cap C=S(x) \cap C \cap B_{0},
$$

hence

$$
u_{\bar{t}} \in \operatorname{int}_{M}\left[S(x) \cap C \cap \bar{B}_{0}\right],
$$


and (2) follows.

Now we can prove the Hausdorff lower semicontinuity of the multifunction $S_{0}$. To this aim, observe that by (2) and Theorem A of [25] (setted in the affine manifold $M=\operatorname{aff}(\mathrm{D})$ by an obvious translation), the multifunction

$$
x \in C \rightarrow S(x) \cap C_{0} \subseteq M
$$

is Hausdorff lower semicontinuous (since $S$ is Hausdorff lower semicontinuous with closed convex values, $C_{0}$ is closed and convex and each set $S(x) \cap C_{0}$ is bounded and has nonempty interior in $M$ ). At this point, the Hausdorff lower semicontinuity of $S_{0}: C_{0} \rightarrow 2^{C_{0}}$ follows at once.

(iii) $/ \operatorname{int}_{\text {aff }\left(\mathrm{C}_{0}\right)}\left(S_{0}(x)\right) \neq \emptyset$ for all $x \in C_{0}$.

To see this, observe that (2) implies, in particular, that $\operatorname{int}_{M}\left(C_{0}\right) \neq \emptyset$. Of course, this implies that $\operatorname{aff}\left(\mathrm{C}_{0}\right)=\operatorname{aff}(\mathrm{C})=\operatorname{aff}(\mathrm{D})=\mathrm{M}$. Consequently, by (2) our claim follows.

$(\mathbf{V}) \prime$ For each $y \in C_{0}$, the set $\left\{x \in C_{0}: \inf _{\varphi \in T(x)}\langle\varphi, x-y\rangle \leq 0\right\}$ is compactly closed.

To see this, observe that, for each fixed $y \in C_{0}$, one has

$$
\left\{x \in C_{0}: \inf _{\varphi \in T(x)}\langle\varphi, x-y\rangle \leq 0\right\}=C_{0} \cap\left\{x \in C: \inf _{\varphi \in T(x)}\langle\varphi, x-y\rangle \leq 0\right\} .
$$

Since $y \in C$ and $C_{0}$ is closed, our claim follows at once by assumption (v).

(Vi) I For each $x \in C_{0} \backslash K$, with $x \in S_{0}(x)$, and each $\varphi \in T(x)$, one has

$$
\sup _{y \in S_{0}(x) \cap H}\langle\varphi, x-y\rangle>0 .
$$

To see this, let $x \in C_{0} \backslash K$, with $x \in S_{0}(x)$, and $\varphi \in T(x)$ be fixed. Since, in particular, $x \in C \backslash K$ and $x \in S(x)$, by assumption (vi) there exists $y^{*} \in S(x) \cap H$ such that $\left\langle\varphi, x-y^{*}\right\rangle>0$. Since $H \subseteq C \cap B_{0}$, it follows that $y^{*} \in C_{0}$, hence $y^{*} \in S_{0}(x) \cap H$. Our claim follows at once.

Step 2. Let $M_{0}$ be the linear subspace of $X$ corresponding to $M$, and observe that $M_{0}$ may not be closed in $X$.

For each $z \in \overline{\mathrm{co}} K$, choose any point $u_{z} \in \operatorname{int}_{M} S_{0}(z)$ (which is nonempty by (iii) $\left.^{\prime}\right)$. By Proposition 2.3, taking into account (ii) ${ }^{\prime}$, there exists an open bounded neighborhood $U_{z}$ of $z$ in $X$ such that

$$
u_{z} \in \operatorname{int}_{M}\left(\bigcap_{v \in U_{z} \cap C_{0}} S_{0}(v)\right) .
$$


Since the set $\overline{\mathrm{co}} K$ is compact, there exist $z_{1}, \ldots, z_{m} \in \overline{\mathrm{co}} K$ such that

$$
\overline{\mathrm{co}} K \subseteq \bigcup_{i=1}^{m}\left(U_{z_{i}} \cap M\right) .
$$

Put

$$
\Sigma_{0}:=\bigcup_{i=1}^{m}\left(U_{z_{i}} \cap M\right) .
$$

Since $M \backslash \Sigma_{0} \neq \emptyset$ (note that $\Sigma_{0}$ is bounded), $M \backslash \Sigma_{0}$ is closed in $M$, and $\overline{\mathrm{co}} K$ is compact, by (7) we get

$$
\xi:=\inf \left\{d\left(a, M \backslash \Sigma_{0}\right): a \in \overline{\mathrm{co}} K\right\}>0 .
$$

If we put

$$
\Sigma:=\overline{\mathrm{co}} K+\left[\bar{B}\left(0_{X}, \frac{\xi}{2}\right) \cap M_{0}\right],
$$

we have that $\Sigma$ is convex and closed in $M$, and also $\Sigma \subseteq \Sigma_{0}$.

Step 3. Let $\mathcal{V}$ be the family of all finite-dimensional linear subspaces of $X$ containing the set

$$
H \cup\left\{u_{z_{1}}, \ldots, u_{z_{m}}\right\} .
$$

At this point, fix $V \in \mathcal{V}$. Put

$$
\Omega:=\overline{C_{0} \cap \Sigma \cap V}
$$

Note that

$$
H \subseteq C_{0} \cap \Sigma \cap V \subseteq \Omega \subseteq C_{0} \cap V .
$$

In particular, $\Omega \neq \emptyset$. Let $S_{V}: \Omega \rightarrow 2^{\Omega}$ be the multifunction defined by setting, for each $x \in \Omega$,

$$
S_{V}(x):=S_{0}(x) \cap \Omega=S_{0}(x) \cap \overline{C_{0} \cap \Sigma \cap V} .
$$

Now, let $J: X^{*} \rightarrow V^{*}$ be the function defined by putting, for each $\varphi \in X^{*}$,

$$
\langle J(\varphi), u\rangle:=\langle\varphi, u\rangle \text { for all } u \in V,
$$

and let $T_{V}: \Omega \rightarrow 2^{V^{*}}$ be the multifunction defined by setting, for each $x \in \Omega$,

$$
T_{V}(x):=J(T(x)) .
$$

Now we want to apply Theorem 4.2(a) of [12] to the finite-dimensional problem $\operatorname{GQVI}\left(\Omega, T_{V}, S_{V}\right)$. To this aim, observe what follows.

(a) The set $\Omega$ is a nonempty closed convex subset of $V$. 
(b) The multifunction $S_{V}: \Omega \rightarrow 2^{\Omega}$ has closed graph and nonempty convex values. Indeed, since $H \subseteq \Omega$, by (i) ${ }^{\prime}$ we have that $S_{V}(x) \neq \emptyset$ for all $x \in \Omega$. Moreover, since $S_{0}$ has closed graph and convex values, by the definition of $S_{V}$ and the convexity of $\Omega$ it follows that $S_{V}$ has closed graph and convex values.

(c) The multifunction $S_{V}: \Omega \rightarrow 2^{\Omega}$ is lower semicontinuous. In order to prove this, we first observe that

$$
\Sigma \cap V \cap \operatorname{int}_{M} S_{0}(x) \neq \emptyset \text { for all } \quad x \in \Omega .
$$

Indeed, let $x \in \Omega$ be fixed, and choose $x^{\prime} \in C_{0} \cap \Sigma \cap V$ such that $\left\|x-x^{\prime}\right\|_{X} \leq \xi / 4$. Hence,

$$
x-x^{\prime} \in M_{0} \cap \bar{B}\left(0_{X}, \frac{\xi}{4}\right) .
$$

Since $x^{\prime} \in \Sigma$, by (8) and (9) it follows that

$$
x \in \overline{\mathrm{co}} K+\left[\bar{B}\left(0_{X}, \frac{3 \xi}{4}\right) \cap M_{0}\right] \subseteq \Sigma_{0} .
$$

Consequently, there is $i \in\{1, \ldots, m\}$ such that $x \in U_{z_{i}}$. By (6), we get in particular that $u_{z_{i}} \in \operatorname{int}_{M} S_{0}(x)$, hence

$$
u_{z_{i}} \in V \cap \operatorname{int}_{M} S_{0}(x) \neq \emptyset .
$$

Fix any $v \in S_{0}(x) \cap H$ (which is nonempty by (i) ${ }^{\prime}$ ). By the convexity of $S_{0}(x)$ we have that

$$
\left.\left.v+t\left(u_{z_{i}}-v\right) \in V \cap \operatorname{int}_{M} S_{0}(x) \text { for all } t \in\right] 0,1\right] .
$$

On the other hand, since by (9) we have

$$
v+\left[\bar{B}\left(0_{X}, \frac{\xi}{2}\right) \cap M_{0}\right] \subseteq \Sigma,
$$

then there exists $\left.\left.t^{\prime} \in\right] 0,1\right]$ such that

$$
\left.v+t\left(u_{z_{i}}-v\right) \in \Sigma \quad \text { for all } \quad t \in\right] 0, t^{\prime}[.
$$

In particular, by (11) and (12) we have

$$
V \cap \Sigma \cap \operatorname{int}_{M} S_{0}(x) \neq \emptyset,
$$

as claimed. Thus, (10) holds. Now we can prove the lower semicontinuity of $S_{V}$. Let $\bar{x} \in \Omega$ and let $W$ be an open set in $M$ such that

$$
S_{V}(\bar{x}) \cap W \neq \emptyset .
$$


By (10), we can choose a point $v$ such that

$$
v \in \Sigma \cap V \cap \operatorname{int}_{M} S_{0}(\bar{x}) \subseteq S_{V}(\bar{x}) .
$$

Fix $\bar{v} \in W \cap S_{V}(\bar{x})$. By the convexity of $S_{0}(\bar{x})$ we have that

$$
\left.\left.\bar{v}+t(v-\bar{v}) \in \Omega \cap \operatorname{int}_{M} S_{0}(\bar{x}) \quad \text { for all } t \in\right] 0,1\right] .
$$

On the other hand, since $W$ is open in $M$, there exists $\vartheta>0$ such that

$$
\bar{v}+\left[\bar{B}\left(0_{X}, \vartheta\right) \cap M_{0}\right] \subseteq W .
$$

Consequently, by (13) and (14), there exists $\tau \in] 0,1]$ such that

$$
\bar{v}+\tau(v-\bar{v}) \in \Omega \cap W \cap \operatorname{int}_{M} S_{0}(\bar{x}) .
$$

By Proposition 2.3, there is a neighborhood $Z_{\bar{x}}$ of $\bar{x}$ in $C_{0}$ such that

$$
\bar{v}+\tau(v-\bar{v}) \in \operatorname{int}_{M}\left(\bigcap_{x \in Z_{\bar{x}}} S_{0}(x)\right) .
$$

By (15) and (16), we get

$$
\bar{v}+\tau(v-\bar{v}) \in \Omega \cap W \cap \operatorname{int}_{M} S_{0}(x) \subseteq W \cap S_{V}(x) \text { for all } \quad x \in Z_{\bar{x}},
$$

hence

$$
S_{V}(x) \cap W \neq \emptyset \quad \text { for all } \quad x \in Z_{\bar{x}}
$$

as desired.

(d) The set $T_{V}(x)$ is nonempty and compact for each $x \in \Omega$, and convex for each $x \in \Omega$, with $x \in S_{V}(x)$. This follows directly from assumption (iv) and the definition of $T_{V}$.

(e) One has $\operatorname{aff}\left(\mathrm{S}_{\mathrm{V}}(\mathrm{x})\right)=\operatorname{aff}(\Omega)$ for all $x \in \Omega$. To see this, fix $x \in \Omega$. Observe that the set

$$
A:=\operatorname{int}_{M} S_{0}(x) \cap \operatorname{aff}(\Omega)
$$

is open in $\operatorname{aff}(\Omega)$ and by $(10)$ one has

$$
\begin{aligned}
\emptyset \neq & \Sigma \cap V \cap \operatorname{int}_{M} S_{0}(x) \\
= & C_{0} \cap \Sigma \cap V \cap \operatorname{int}_{M} S_{0}(x) \\
& \subseteq \Omega \cap \operatorname{int}_{M} S_{0}(x) \\
= & \Omega \cap \operatorname{aff}(\Omega) \cap \operatorname{int}_{M} S_{0}(\mathrm{x}) \\
= & A \cap \Omega,
\end{aligned}
$$


hence $A \cap \Omega \neq \emptyset$. Consequently, by Proposition 2.1 of [12] (setted in the affine manifold aff $(\Omega)$ by an obvious translation), we get

$$
\operatorname{aff}(\mathrm{A} \cap \Omega)=\operatorname{aff}(\Omega) .
$$

Since

$$
A \cap \Omega \subseteq S_{V}(x) \subseteq \Omega
$$

by (17) we get our claim.

(f) For each $y \in \Omega$, the set

$$
\left\{x \in \Omega: \inf _{\tilde{\varphi} \in T_{V}(x)}\langle\tilde{\varphi}, x-y\rangle \leq 0\right\}
$$

is closed. Indeed, if we fix $y \in \Omega$, we have

$$
\begin{aligned}
\left\{x \in \Omega: \inf _{\tilde{\varphi} \in T_{V}(x)}\langle\tilde{\varphi}, x-y\rangle \leq 0\right\} & =\left\{x \in \Omega: \inf _{\varphi \in T(x)}\langle\varphi, x-y\rangle \leq 0\right\} \\
& =\left\{x \in C_{0}: \inf _{\varphi \in T(x)}\langle\varphi, x-y\rangle \leq 0\right\} \cap \Omega,
\end{aligned}
$$

which is closed by (v) (taking into account that $\Omega$ is closed, $\Omega \subseteq V$ and $V$ is finite-dimensional).

(g) The set $K_{\Omega}:=K \cap \Omega$ is compact and

$$
S_{V}(x) \cap K_{\Omega} \neq \emptyset \text { for all } x \in \Omega
$$

(taking into account that $H \subseteq \Omega \cap K$, this follows easily by (i) ${ }^{\prime}$ and the definition of $S_{V}$ ).

(h) For each fixed $x \in \Omega \backslash K_{\Omega}$, with $x \in S_{V}(x)$, and each fixed $\tilde{\varphi} \in T_{V}(x)$, there exists $y \in S_{V}(x) \cap K_{\Omega}$ such that $\langle\tilde{\varphi}, x-y\rangle>0$. Indeed, let $x$ and $\tilde{\varphi}$ be as above, and let $\varphi \in T(x)$ be such that $\tilde{\varphi}=J(\varphi)$. By the definitions of $K_{\Omega}$ and $S_{V}$ one has that $x \in C_{0} \backslash K$ and $x \in S_{0}(x)$. Therefore, by (vi)', there exists a point

$$
y \in S_{0}(x) \cap H \subseteq S_{V}(x) \cap K_{\Omega}
$$

such that $\langle\varphi, x-y\rangle>0$. Since $x, y \in V$, then we have $\langle\tilde{\varphi}, x-y\rangle>0$, as desired.

Thus, all the assumptions of Theorem 4.2(a) of [12] are satisfied. Consequently, there exists a pair $\left(x_{V}, \tilde{\varphi}_{V}\right) \in \Omega \times V^{*}$ such that

(18) $x_{V} \in S_{V}\left(x_{V}\right), \quad \tilde{\varphi}_{V} \in T_{V}\left(x_{V}\right) \quad$ and $\quad\left\langle\tilde{\varphi}_{V}, x_{V}-y\right\rangle \leq 0 \quad \forall y \in S_{V}\left(x_{V}\right)$.

Let $\varphi_{V} \in T\left(x_{V}\right)$ be such that $\tilde{\varphi}_{V}=J\left(\varphi_{V}\right)$. By (18) we get

$$
\left\langle\varphi_{V}, x_{V}-y\right\rangle \leq 0 \text { for all } y \in S_{0}\left(x_{V}\right) \cap \Omega .
$$


By (19) and (vi)', taking into account that $H \subseteq \Omega$, we have that $x_{V} \in K$. We now prove that

$$
\left\langle\varphi_{V}, x_{V}-y\right\rangle \leq 0 \quad \text { for all } y \in S_{0}\left(x_{V}\right) \cap V .
$$

Indeed, if $y \in S_{0}\left(x_{V}\right) \cap V$ is fixed, since

$$
\begin{gathered}
x_{V} \in K \subseteq \overline{\mathrm{co}} K \subseteq C_{0} \subseteq M, \\
y \in S_{0}\left(x_{V}\right) \subseteq C_{0} \subseteq M, \\
M-M \subseteq M_{0},
\end{gathered}
$$

and $C_{0}$ is convex, we have that

$$
x_{V}+t\left(y-x_{V}\right) \in C_{0} \cap\left[\overline{\mathrm{co}} K+\left(\bar{B}\left(0_{X}, \frac{\xi}{2}\right) \cap M_{0}\right)\right]=C_{0} \cap \Sigma
$$

for a sufficiently small $t \in] 0,1\left[\right.$. Hence, by the convexity of $S_{0}\left(x_{V}\right)$ and by the definition of $\Omega$, we have

$$
x_{V}+t\left(y-x_{V}\right) \in C_{0} \cap \Sigma \cap V \cap S_{0}\left(x_{V}\right) \subseteq \Omega \cap S_{0}\left(x_{V}\right) .
$$

By (19) we get

$$
0 \geq\left\langle\varphi_{V}, x_{V}-\left(x_{V}+t\left(y-x_{V}\right)\right)\right\rangle=t\left\langle\varphi_{V}, x_{V}-y\right\rangle,
$$

hence (20) is proved.

Resuming, we have proved that for each $V \in \mathcal{V}$ there exists a pair $\left(x_{V}, \varphi_{V}\right) \in$ $(K \cap V) \times X^{*}$ such that

(21) $x_{V} \in S_{0}\left(x_{V}\right), \quad \varphi_{V} \in T\left(x_{V}\right) \quad$ and $\quad\left\langle\varphi_{V}, x_{V}-y\right\rangle \leq 0 \quad \forall y \in S_{0}\left(x_{V}\right) \cap V$.

Step 4. Now we consider the net $\left\{x_{V}\right\}_{V \in \mathcal{V}}$, with $\mathcal{V}$ ordered by the ordinary set inclusion $\subseteq$. Since $K$ is compact, the net $\left\{x_{V}\right\}_{V \in \mathcal{V}}$ has a cluster point $\hat{x} \in K$. Since the multifunction $S_{0}$ has closed graph, by (21) we get $\hat{x} \in S_{0}(\hat{x})$. Moreover, by (iii)' we have that $\operatorname{int}_{M} S_{0}(\hat{x}) \neq \emptyset$. We now claim that

$$
\inf _{\varphi \in T(\hat{x})}\langle\varphi, \hat{x}-y\rangle \leq 0 \quad \text { for all } \quad y \in \operatorname{int}_{M} S_{0}(\hat{x}) .
$$

On the contrary, assume that there exists $\tilde{y} \in \operatorname{int}_{M} S_{0}(\hat{x})$ such that

$$
\inf _{\varphi \in T(\hat{x})}\langle\varphi, \hat{x}-\tilde{y}\rangle>0 .
$$

By Proposition 2.3, there exists $\sigma>0$ such that

$$
\tilde{y} \in \operatorname{int}_{M}\left(\bigcap_{x \in B(\hat{x}, \sigma) \cap C_{0}} S_{0}(x)\right) .
$$


By (23) and (v)', since the set

$$
\left\{x \in K: \inf _{\varphi \in T(x)}\langle\varphi, x-\tilde{y}\rangle>0\right\}
$$

is open in $K$, there exists $\alpha \in] 0, \sigma$ [ such that

$$
\inf _{\varphi \in T(x)}\langle\varphi, x-\tilde{y}\rangle>0 \text { for all } \quad x \in B(\hat{x}, \alpha) \cap K .
$$

By construction, there exists $\hat{V} \in \mathcal{V}$ such that $\tilde{y} \in \hat{V}$ and $x_{\hat{V}} \in \bar{B}(\hat{x}, \alpha)$. By (24) we get $\tilde{y} \in S_{0}\left(x_{\hat{V}}\right) \cap \hat{V}$. Consequently, (21) implies that

$$
\left\langle\varphi_{\hat{V}}, x_{\hat{V}}-\tilde{y}\right\rangle \leq 0 .
$$

On the other hand, (25) implies that

$$
\inf _{\varphi \in T\left(x_{\hat{V}}\right)}\left\langle\varphi, x_{\hat{V}}-\tilde{y}\right\rangle>0
$$

hence, in particular,

$$
\left\langle\varphi_{\hat{V}}, x_{\hat{V}}-\tilde{y}\right\rangle>0
$$

which contradicts (26). Hence, (22) holds.

Applying Theorem 5 at p. 216 of [1], and taking into account (ii)', assumption (iv) and the inequality (22), it follows that there exists $\hat{\varphi} \in T(\hat{x})$ such that

$$
\begin{aligned}
\sup _{y \in \operatorname{int}_{M} S_{0}(\hat{x})}\langle\hat{\varphi}, \hat{x}-y\rangle & =\inf _{\varphi \in T(\hat{x})} \sup _{y \in \operatorname{int}_{M} S_{0}(\hat{x})}\langle\varphi, \hat{x}-y\rangle \\
& =\sup _{y \in \operatorname{int}_{M} S_{0}(\hat{x})} \inf _{\varphi \in T(\hat{x})}\langle\varphi, \hat{x}-y\rangle \leq 0 .
\end{aligned}
$$

Of course, this implies

$$
\sup _{y \in S_{0}(\hat{x})}\langle\hat{\varphi}, \hat{x}-y\rangle \leq 0
$$

We now prove that

$$
\langle\hat{\varphi}, \hat{x}-y\rangle \leq 0 \quad \text { for all } \quad y \in S(\hat{x}) \cap C .
$$

To this aim, let $y^{\prime}$ be any point in $S(\hat{x}) \cap C$. Since $\hat{x} \in K \subseteq B_{0}$, and $B_{0}$ is open in $X$, we have that

$$
w_{t}:=\hat{x}+t\left(y^{\prime}-\hat{x}\right) \in S(\hat{x}) \cap C \cap B_{0} \subseteq S_{0}(\hat{x})
$$

for sufficiently small $t \in] 0,1[$. By (27), for such $t$ we have

$$
0 \geq\left\langle\hat{\varphi}, \hat{x}-w_{t}\right\rangle=t\left\langle\hat{\varphi}, \hat{x}-y^{\prime}\right\rangle
$$


as desired. Hence, (28) holds and the first part of the conclusion is proved.

Finally, we prove the last part of the conclusion. To this aim, assume that $\hat{x} \in \operatorname{int}_{M}(C)$, and fix any $y^{*} \in S(\hat{x})$. Since $\hat{x} \in \operatorname{int}_{M}(C)$, there exists $\varepsilon>0$ such that

$$
\hat{x}+\left[\bar{B}(0, \varepsilon) \cap M_{0}\right] \subseteq C .
$$

Since $y^{*} \in S(\hat{x}) \subseteq D \subseteq M, M-M \subseteq M_{0}$, and $S(\hat{x})$ is convex, by (29) we have that

$$
v_{\tau}:=\hat{x}+\tau\left(y^{*}-\hat{x}\right) \in C \cap S(\hat{x})
$$

for sufficiently small $\tau \in] 0,1[$. By (28), for such $\tau$ we have

$$
0 \geq\left\langle\hat{\varphi}, \hat{x}-v_{\tau}\right\rangle=\tau\left\langle\hat{\varphi}, \hat{x}-y^{*}\right\rangle,
$$

as desired. The proof is now complete.

\section{An ApPlication}

We now present an application of Theorem 1.2. First of all, following the argument announced in the introduction, we prove the following result (as before, we put $\left.S_{C}(x):=S(x) \cap C\right)$.

Theorem 4.1. Let $\left(X,\|\cdot\|_{X}\right)$ be a real Banach space with topological dual $X^{*}$, let $C \subseteq D \subseteq X$ be closed convex sets, and let $H$ be a finite-dimensional compact subset of $C$. Let $S: C \rightarrow 2^{D}$ and $T: C \rightarrow 2^{X^{*}}$ be two multifunctions such that assumptions (i)-(v) of Theorem 1.2 are satisfied. Moreover, assume that the set

$$
\operatorname{Fix}(S):=\{x \in C: x \in S(x)\}
$$

is compact. Then, there exists $(\hat{x}, \hat{\varphi}) \in C \times X^{*}$ which solves $\mathrm{GQVI}\left(C, T, S_{C}\right)$. Moreover, if $\hat{x} \in \operatorname{ri}(C)$, then $(\hat{x}, \hat{\varphi})$ solves $\operatorname{EGQVI}(C, D, T, S)$.

Proof. Put $K:=H \cup \operatorname{Fix}(S)$, and observe that the set $K$ is compact and also $K \subseteq C$. At this point, it is immediate to check that assumption (vi) of Theorem 1.2 is satisfied, since

$$
(C \backslash K) \cap \operatorname{Fix}(S)=\emptyset .
$$

Consequently, our conclusion follows at once by Theorem 1.2, taking into account that by (iii) one has $\operatorname{aff}(\mathrm{C})=\operatorname{aff}(\mathrm{D})$, hence $\operatorname{int}_{\mathrm{aff}(\mathrm{D})}(C)=\operatorname{ri}(C)$.

In the particular case where $S(C) \subseteq C$, Theorem 4.1 gives the following existence result for the classical $\operatorname{GQVI}(C, T, S)$ problem, which we state explicitly for further use. 
Corollary 4.2. Let $\left(X,\|\cdot\|_{X}\right)$ be a real Banach space with topological dual $X^{*}, C \subseteq X$ a closed convex sets, $H$ a finite-dimensional compact subset of $C$. Let $S: C \rightarrow 2^{C}$ and $T: C \rightarrow 2^{X^{*}}$ be two multifunctions. Assume that:

(i) $S(x) \cap H \neq \emptyset$ for all $x \in C$;

(ii) the multifunction $S$ is Hausdorfflower semicontinuous with closed graph and convex values;

(iii) $\operatorname{int}_{\mathrm{aff}(\mathrm{C})}(S(x)) \neq \emptyset$ for all $x \in C$;

(iv) $T(x)$ is nonempty and weakly-star compact for each $x \in C$, and convex for each $x \in C$, with $x \in S(x)$;

(v) for each $y \in C$, the set $\left\{x \in C: \inf _{\varphi \in T(x)}\langle\varphi, x-y\rangle \leq 0\right\}$ is compactly closed;

(vi) the set $\operatorname{Fix}(S)$ is compact.

Then there exists $(\hat{x}, \hat{\varphi}) \in C \times X^{*}$ which solves $\operatorname{GQVI}(C, T, S)$.

As an application of the latter result, we now prove the following theorem of the alternative, which extends a recent finite-dimensional result (Theorem 3.1 of [10]) to infinite-dimensional setting.

Theorem 4.3. Let $X$ be a real separable Hilbert space with scalar product $\langle\cdot, \cdot\rangle$, let $C \subseteq X$ be a closed convex set whose affine hull aff( $(\mathrm{C})$ is closed in $X$, and let $S: C \rightarrow 2^{C}$ be a multifunction. Assume that:

(i) the multifunction $S$ is Hausdorfflower semicontinuous with closed graph and convex values;

(ii) $\operatorname{int}_{\mathrm{aff}(\mathrm{C})}(S(x)) \neq \emptyset$ for all $x \in C$;

(iii) there exists a finite-dimensional compact set $H \subseteq C$ such that $S(x) \cap H \neq \emptyset$ for all $x \in C$.

Then, at least one of the following assertions holds:

(A) There exists $x_{0} \in X$ such that $S(x) \equiv C=\left\{x_{0}\right\}$.

(B) The set $\operatorname{Fix}(S):=\{x \in C: x \in S(x)\}$ is not totally bounded.

(C) There exists $\hat{x} \in C$ such that $\hat{x} \in \partial_{r} S(\hat{x})$.

Proof. Let $H_{0}:=\overline{\mathrm{co}}(H)$. By Theorem 6 at p.416 of [19], the set $H_{0}$ is compact. Moreover, we have that $H_{0} \subseteq C$. Let us consider the multifunction $S: H_{0} \rightarrow 2^{H_{0}}$ defined by putting, for each $x \in H_{0}$,

$$
S_{0}(x)=S(x) \cap H_{0} .
$$

By assumption (i), it follows that the multifunction $S_{0}$ has closed graph. Consequently, taking into account (iii), the compactness of $H_{0}$ and Theorem 7.1.16 of 
[24], the multifunction $S_{0}$ is upper semicontinuous with nonempty compact convex values. Therefore, by the classical Fan-Kakutani fixed-point theorem, there exists $x^{*} \in H_{0}$ such that $x^{*} \in S_{0}\left(x^{*}\right) \subseteq S\left(x^{*}\right)$, hence the set $\operatorname{Fix}(S)$ is nonempty. Moreover, observe that $\operatorname{Fix}(S)$ is closed by (i).

Now, assume that assertions (A) and (B) do not hold. Therefore, the set $\operatorname{Fix}(S)$ is compact and $C$ contains more than one point. Let $M:=\operatorname{aff}(\mathrm{C})$, and let $M_{0}$ be the linear subspace associated to $M$ (note that $M_{0}$ is closed since $M$ is closed by assumption). Since $C$ contains more than one point, then $M_{0} \neq\left\{0_{X}\right\}$. Choose any point $z \in M_{0} \backslash\left\{0_{X}\right\}$. We claim that

$$
\sup _{v \in S(x)-S(x)}\langle z, v\rangle>0 \text { for all } x \in C .
$$

To this aim, fix $x \in C$. By (ii), there exists $u \in C$ such that

$$
u \in \operatorname{int}_{M}(S(x)) \text {. }
$$

Since $M_{0}=\operatorname{span}(C-u), M=u+M_{0}$, and $S(x)-S(x) \subseteq M_{0}$, then we get

$$
0_{X} \in \operatorname{int}_{M_{0}}(S(x)-u) \subseteq \operatorname{int}_{M_{0}}(S(x)-S(x)) .
$$

Consequently, there exists $\varepsilon>0$ be such that

$$
B\left(0_{X}, \varepsilon\right) \cap M_{0} \subseteq S(x)-S(x) .
$$

Then, we have that $t z \in S(x)-S(x)$ for sufficiently small $t>0$. Since $\langle z, t z\rangle>0$, the claim (30) follows.

Let $\Phi: C \rightarrow 2^{M_{0}}$ be the multifunction defined by putting, for each $x \in C$,

$$
\Phi(x):=[S(x)-S(x)] \cap\left\{v \in M_{0}:\langle z, v\rangle>0\right\} .
$$

By (30), and taking into account that $S(x)-S(x) \subseteq M_{0}$, we have that the multifunction $\Phi$ has nonempty convex values. Moreover, observe that by Theorems 7.3.11 and 7.3.15 of [24] the multifunction

$$
x \in C \rightarrow S(x)-S(x)
$$

is lower semicontinuous. Since the set $\left\{v \in M_{0}:\langle z, v\rangle>0\right\}$ is open in $M_{0}$, the multifunction $\Phi$ is lower semicontinuous. We now show that

$$
\operatorname{int}_{M_{0}}(\Phi(x)) \neq \emptyset \quad \text { for all } \quad x \in C .
$$

To this aim, fix $x \in C$. Observe that by (31) the set $S(x)-S(x)$ has nonempty interior in $M_{0}$, hence by convexity we get

$$
\left\{v \in M_{0}:\langle z, v\rangle>0\right\} \cap \operatorname{int}_{M_{0}}(S(x)-S(x)) \neq \emptyset .
$$


Since the above set is open in $M_{0}$ and it is contained in $\Phi(x)$, the claim follows. Consequently, since $M_{0}$ is a separable Banach space, by Theorem 2.1 the multifunction $\Phi$ admits a continuous selection. That is, there exists a (single-valued) function $f: C \rightarrow M_{0}$ such that $f(x) \in \Phi(x)$ for each $x \in C$.

Applying Corollary 4.2 (with $T(x)=\{f(x)\}$ ), we have that there exists a point $\hat{x} \in C$ such that

$$
\hat{x} \in S(\hat{x}) \quad \text { and } \quad\langle f(\hat{x}), \hat{x}-y\rangle \leq 0 \quad \text { for all } \quad y \in S(\hat{x}) .
$$

Now, observe that by (ii) we have that $\operatorname{aff}(\mathrm{S}(\hat{\mathrm{x}}))=\mathrm{M}$, hence

$$
\operatorname{ri}(S(\hat{x}))=\operatorname{int}_{M}(S(\hat{x})) \quad \text { and } \quad \partial_{r}(S(\hat{x}))=\partial_{M}(S(\hat{x})) .
$$

In particular, $\operatorname{ri}(S(\hat{x})) \neq \emptyset$.

Now, assume that $\hat{x} \in \operatorname{int}_{M}(S(\hat{x}))=\operatorname{ri}(S(\hat{x}))$. By Proposition 2.2, we get

$$
f(\hat{x}) \in(S(\hat{x})-S(\hat{x}))^{\perp} .
$$

Since

$$
f(\hat{x}) \in \Phi(\hat{x}) \subseteq S(\hat{x})-S(\hat{x}),
$$

it follows that $f(\hat{x})=0_{X}$. On the other hand, since

$$
f(\hat{x}) \in \Phi(\hat{x}) \subseteq\left\{v \in M_{0}:\langle z, v\rangle>0\right\},
$$

we get $f(\hat{x}) \neq 0_{X}$, a contradiction. Such a contradiction implies that $\hat{x} \in \partial_{r}(S(\hat{x}))$, as desired. The proof is complete.

Finally, we observe that Theorem 1.3 follows at once from Theorem 4.3.

\section{ACKNOWLEDGMENTS}

The research of second author was partially supported by the Grant NSC 982923-E-110-003-MY3.

\section{REFERENCES}

1. Mathematical Methods of Game and Economic Theory, North-Holland, Amsterdam, Holland, 1979.

2. C. Baiocchi and A. Capelo, Variational and Quasivariational Inequalities: Application to Free-Boundary Problems, John Wiley and Sons, New York, 1984.

3. A. Bensoussan and J. L. Lions, Nouvelle formulation de problemes de contrôle impulsionnel et applications, C. R. Acad. Sci. Paris, 276 (1973), 1189-1192. 
4. A. Bensoussan, M. Goursat and J. L. Lions, Contrôle impulsionnel et inéquations quasi variationnelles stationnaires, C. R. Acad. Sci. Paris, 276 (1973), 1279-1284.

5. A. Bensoussan and J. L. Lions, Nouvelles méthodes en contrôle impulsionnel, Appl. Math. Optim., 1 (1974), 289-312.

6. D. Chan and J. S. Pang, The generalized quasi-variational inequality problem, Math. Oper. Res., 7 (1982), 211-222.

7. L. J. Chu and C. Y. Lin, On discontinuous quasi-variational inequalities, Discuss. Math. Differ. Incl. Control Optim., 27 (2007), 199-212.

8. P. Cubiotti, Finite-dimensional quasi-variational inequalities associated with discontinuous functions, J. Optim. Theory Appl., 72 (1992), 577-582.

9. P. Cubiotti, An existence theorem for generalized quasi-variational inequalities, SetValued Anal., 1 (1993), 81-87.

10. P. Cubiotti, Application of quasi-variational inequalities to linear control systems, $J$. Optim. Theory Appl., 89 (1996), 101-113.

11. P. Cubiotti, Generalized quasi-variational inequalities in infinite-dimensional normed spaces, J. Optim. Theory Appl., 92 (1997), 457-475.

12. P. Cubiotti, Generalized quasi-variational inequalities without continuities, J. Optim. Theory Appl., 92 (1997), 477-495.

13. P. Cubiotti, On the discontinuous infinite-dimensional generalized quasivariational inequality problem, J. Optim. Theory Appl., 115 (2002), 97-111.

14. P. Cubiotti and J. C. Yao, Discontinuous implicit quasi-variational inequalities with applications to fuzzy mappings, Math. Methods Oper. Res., 46 (1997), 213-228.

15. P. Cubiotti and X. Z. Yuan, A generalised quasi-variational inequality without upper semicontinuity, Bull. Austral. Math. Soc., 54 (1996), 247-254.

16. M. De Luca and A. Maugeri, Quasi-variational inequalities and applications to equilibrium problems with elastic demand, in: Nonsmooth Optimization and Related Topics (F. H. Clarke, V. F. Dem'yanov and F. Giannessi, eds.), Ettore Majorana International Science Series, Plenum Press, 1989.

17. M. De Luca and A. Maugeri, Discontinuous quasi-variational inequalities and applications to equilibrium problems, in: Nonsmooth Optimization. Methods and Applications, (F. Giannessi, ed.), Gordon and Breach Sc. Publ., 1992, pp. 70-74.

18. M. De Luca, Generalized quasi-variational inequalities and traffic equilibrium problem, in: Variational Inequalities and Network Equilibrium Problems, (F. Giannessi and A. Maugeri, eds.), Plenum Press, New York, 1995.

19. N. Dunford and J. T. Schwartz, Linear Operators, Part I, New York, NY, 1958.

20. P. T. Harker, A variational inequality approach for the determination of oligopolistic market equilibrium, Math. Programming, 30 (1984), 105-111. 
21. P. T. Harker, Generalized Nash games and quasi- variational inequalities, European J. Oper. Res., 54 (1991), 81-94.

22. P. T. Harker and J. S. Pang, Finite-dimensional variational inequality and nonlinear complementarity problems: A survey of theory, algorithms and applications, Math. Programming, 48 (1990), 161-220.

23. P. Hartman and G. Stampacchia, On some nonlinear elliptic differential functional equations, Acta Math., 115 (1966), 153-188.

24. E. Klein and A. C. Thompson, Theory of Correspondences, John Wiley and Sons, New York, 1984.

25. A. Lechicki and A. Spakowski, A note on intersection of lower semicontinuous multifunctions, Proc. Amer. Math. Soc., 95 (1985), 119-122.

26. M. L. Lunsford, Generalized variational and quasi-variational inequalities with discontinuous operators, J. Math. Anal. Appl., 214 (1997), 245-263.

27. A. Maugeri, Convex programming, variational inequalities and applications to the traffic equilibrium problem, Appl. Math. Optim., 16 (1987), 169-185.

28. E. Michael, Continuous selections I, Ann. of Math., 63 (1956), 361-382.

29. B. Riccert, Un theoreme d'existence pour les inequations variationelles, C. R. Acad. Sci. Paris Sér. I Math., 301 (1985), 885-888.

30. B. Riccert, Basic existence theorems for generalized variational and quasi-variational inequalities, in Variational Inequalities and Network Equilibrium Problems, (F. Giannessi and A. Maugeri, eds.), Plenum Press, New York, 1995.

31. M. H. Shih and K. K. Tan, Generalized quasi-variational inequalities in locally convex topological vector spaces, J. Math. Anal. Appl., 108 (1985), 333-343.

32. E. Tarafdar and X. Z. Yuan, Non-compact generalized quasi-variational inequalities in locally convex topological vector spaces, Nonlin. World, 1 (1994), 373-383.

33. J. C. Yao, The generalized quasi-variational inequality problem with applications, $J$. Math. Anal. Appl., 158 (1991), 139-160.

34. J. C. Yao, Generalized quasi-variational inequality problems with discontinuous mappings, Math. Oper. Res., 20 (1995), 465-478.

35. J. C. Yao and J. S. Guo, Variational and generalized variational inequalities with discontinuous mappings, J. Math. Anal. Appl., 182 (1994), 371-392.

36. N. D. Yen, On a class of discontinuous vector-valued functions and the associated quasi-variational inequalities, Optimization, 30 (1994), 197-203.

37. N. D. Yen, On an existence theorem for generalized quasi-variational inequalities, Set-Valued Anal., 3 (1995), 1-10. 


\section{Paolo Cubiotti}

Department of Mathematics

University of Messina

Viale F. Stagno d'Alcontres 31

98166 Messina

Italy

E-mail: cubiotti@dipmat.unime.it

Jen-Chih Yao

Center for General Education

Kaohsiung Medical University

Kaohsiung 80707, Taiwan

E-mail: yaojc@kmu.edu.tw 